\title{
Multivariate Quality Loss Model and its Coefficient Determination
}

\author{
Shuhai Fan, Xia Cao \\ Department of Industrial Engineering, Nanjing University of Technology, Nanjing, China. \\ Email: fanshuhai@tsinghua.org.cn
}

Received August 28 $8^{\text {th }}, 2009$; revised October $10^{\text {th }}, 2009$; accepted November $27^{\text {th }}, 2009$.

\begin{abstract}
In the early 1970s, based on single index deducted from absolute quality deviants, Genichi Taguchi proposed the quality loss module. This module builds the foundation of his three-stage design theory, e.g. system design, parameter design and tolerance design. In actual production process, nevertheless, it is multiple quality indices that influence the total quality. Consequently, the interaction of the quality indices should be imported into the module as a key factor. Accordingly, based on several indices of relative quality deviation, introduce a 2-order multivariate quality module at first. Next, extend the module to 3 or even higher orders. Then, improve the previous quality module by simplifying the 2-order module as a multivariate quality loss module. Finally, bring forward a significant solution to determinate all the coefficients in the multivariate quality loss module and describe its work flow as well.
\end{abstract}

Keywords: Quality Loss, Multiple Variables, Model Building

\section{Introduction}

In the early 1970s, Dr. Genichi Taguchi, the famous quality management expert of Japan, carried on an innovation research into the theories and methods of quality management [1]. He established the famous "Taguchi Three-stage Design Methods", the system design, the parameter design and the tolerance design. The core of his theory was his quality loss model $[2,3]$. And the quality loss model was based on the absolute quality deviant, which suited for the single quality index. However, in the actual production process, many partial quality indices often affected the total quality in a coactions mode. After these interactions being considered, a multivariate quality loss mode can be brought forward [4].

To this many partial quality indices, Chan and Ibrahim studied a quality evaluation model using loss function for multiple S-type quality characteristics [5]. To determinate the coefficients of the multivariate quality loss mode, AHP method or tolerance method can be used $[6,7]$.

\section{Model Building}

The total quality is a synthetic quality, which is often a coaction of some machining qualities and some assembling qualities of every part [1]. And it can be regarded as a function of every partial quality (including the machining qualities and the assembling qualities):

$$
Q_{\text {product }}=f\left(Q_{1}, Q_{2}, \ldots Q_{h} \ldots, Q_{H}\right)
$$

Assuming that $f$ has continuous partial derivatives till 3 -order in a neighborhood $D$ of the origin, $\mathrm{P}_{0}(0,0 \ldots 0)$.

There are $H$ partial quality indices altogether, $q_{1}, q_{2}, \ldots$ $q_{\mathrm{H}}$. These partial quality indices are all continuous types; their values are very small and stand for relative quality deviants. When the values of $q_{1}, q_{2}, \ldots q_{\mathrm{H}}$ equal 0 , it stands for no quality deviant (Here we use the relative quality deviants to get dimensionless. Thus a minor error can be avoided in the derivation process of Taguchi's model.) [8]. Then the product quality can be expressed as follows:

$$
\begin{aligned}
f\left(q_{1}, q_{2} \ldots, q_{h} \ldots, q_{H}\right)= & f(0,0 \ldots, 0)+\sum_{h=1}^{H} f_{Q_{h}}^{\prime}(0,0 \ldots, 0) q_{h} \\
& +\frac{1}{2 !} \sum_{h_{1}, h_{2}=1}^{H} f_{Q_{h_{1}} Q_{h_{2}}}^{\prime \prime}(0,0 \ldots, 0) q_{h_{1}} q_{h_{2}}+R_{2}
\end{aligned}
$$

while, $R_{2}$ is the 2-order remainder term.

$$
\begin{gathered}
R_{2}=\frac{1}{3 !} \sum_{h_{1}, h_{2}, h_{3}=1}^{H} f_{Q_{h_{1}}}^{\prime \prime \prime} Q_{h_{2}} Q_{h_{3}} \\
\left(0 q_{1} \ldots \theta q_{h} \ldots \theta q_{H}\right) q_{h_{1}} q_{h_{2}} q_{h_{3}} \\
(0<\theta<1)
\end{gathered}
$$

A brief proof is as follows:

considering a single variant function $Q(t)$

$$
Q(t)=f\left(q_{1} t, q_{2} t \ldots, q_{h} t \ldots, q_{H} t\right), \quad(-1 \leq t \leq 1)
$$


$Q(t)$ has continuous partial derivatives till 3-order for $(-1 \leq t \leq 1)$.

Obeying the 2-order single variant Talylor's formula with Lagrange remainder term, we get:

$$
Q(t)=Q(0)+Q^{\prime}(0) t+\frac{1}{2 !} Q^{\prime \prime}(0) t^{2}+\frac{1}{3 !} Q^{\prime \prime \prime}(\theta t) t^{3},(0<\theta<1)
$$

Let $\mathrm{t}=1$, we get

$$
Q(1)=Q(0)+Q^{\prime}(0)+\frac{1}{2 !} Q^{\prime \prime}(0)+\frac{1}{3 !} Q^{\prime \prime \prime}(\theta)
$$

Follow the definition of $Q(t)$ and the differential method of composite function. We get

$$
\begin{gathered}
Q^{\prime}(t)=\sum_{h=1}^{H} f_{Q_{h}}^{\prime}\left(q_{1} t, \ldots q_{h} t, \ldots, q_{H} t\right) q_{h} \\
Q^{\prime \prime}(t)=\sum_{h_{1}, h_{2}=1}^{H} f_{Q_{h_{1}} Q_{h_{2}}}^{\prime \prime}\left(q_{1} t, \ldots q_{h} t, \ldots, q_{H} t\right) q_{h_{1}} q_{h_{2}} \\
Q^{\prime \prime \prime}(t)=\sum_{h_{1}, h_{2}, h_{3}=1}^{H} f_{Q_{h_{1}} Q_{h_{2}} Q_{h_{3}}}^{\prime \prime}\left(q_{1} t, \ldots q_{h} t, \ldots, q_{H} t\right) q_{h_{1}} q_{h_{2}} q_{h_{3}}
\end{gathered}
$$

Then, when $t=0$,

$$
\begin{gathered}
Q^{\prime}(0)=\sum_{h=1}^{H} f_{Q_{h}}^{\prime}(0, \ldots 0, \ldots, 0) q_{h} \\
Q^{\prime \prime}(0)=\sum_{h_{1}, h_{2}=1}^{H} f_{Q_{h_{1}} Q_{h_{2}}}^{\prime \prime}(0, \ldots 0, \ldots, 0) q_{h_{1}} q_{h_{2}} \\
Q^{\prime \prime \prime}(0)=\sum_{h_{1}, h_{2}, h_{3}=1}^{H} f_{Q_{h_{1}} Q_{h_{2}} Q_{h_{3}}}^{\prime \prime}(0, \ldots 0, \ldots, 0) q_{h_{1}} q_{h_{2}} q_{h_{3}}
\end{gathered}
$$

while

$$
\begin{gathered}
Q(1)=f\left(q_{1}, q_{2} \ldots, q_{h} \ldots, q_{H}\right), \\
Q(0)=f(0, \ldots, 0 \ldots, 0)
\end{gathered}
$$

Substitute the results into (1)

The expansion equation can get proved.

To estimate the error term, we have:

$f$ has continuous partial derivatives till 3-order; in the neighborhood of $(0, \ldots, 0, \ldots 0)$; the 3 -order partial derivative has a bound $\mathrm{M}$.

denote

$$
\rho=\sqrt{q_{1}^{2}+q_{2}^{2}+\ldots q_{H}^{2}}=\sqrt{\sum_{h=1}^{H} q_{h}^{2}}, \quad(\rho \rightarrow 0)
$$

then

$$
\begin{aligned}
& \left|R_{2}\right| \leq\left|\frac{M}{3 !} \sum_{h_{1}, h_{2}, h_{3}=1}^{H} q_{h_{1}} q_{h_{2}} q_{h_{3}}\right|=\left|\frac{M}{3 !}\left(q_{1}+q_{2}+\ldots+q_{H}\right)^{3}\right| \\
& =\frac{M}{3 !}\left[\sqrt{\left(q_{1}+q_{2}+\ldots+q_{H}\right)^{2}}\right]^{3}=\frac{M}{3 !}\left[\sqrt{\sum_{h_{1}, h_{2}=1}^{H} q_{h_{1}} q_{h_{2}}}\right]^{3}
\end{aligned}
$$

$$
\begin{aligned}
& \leq \frac{M}{3 !}\left[\sqrt{\sum_{h_{1}, h_{2}=1}^{H} \frac{1}{2}\left(q_{h_{1}}{ }^{2}+q_{h_{2}}{ }^{2}\right)}\right]^{3} \\
& =\frac{M}{3 !}\left[\sqrt{H\left(q_{1}{ }^{2}+q_{2}{ }^{2}+\ldots+q_{H}{ }^{2}\right)}\right]^{3} \\
= & \frac{M}{3 !}(\sqrt{H})^{3}\left[\sqrt{\sum_{h=1}^{H} q_{h}{ }^{2}}\right]^{3}=\frac{M}{3 !}(\sqrt{H})^{3} \rho^{3}
\end{aligned}
$$$$
\therefore R_{2}=o\left(\rho^{2}\right), \quad(\rho \rightarrow 0)
$$

It is the general form that is used to describe the total quality index in the above formula. If the quality loss form index is adopted, the model can be much more simplified. Imply that when all the partial quality indices equal 0 , the total quality index takes the minimum, 0 . It is easy to see that the 0 -order and 1 -order derivative can be gotten rid of. Then a new product quality loss model can be built as follows:

$$
L=Q_{\text {product }}=\sum_{h=1}^{H} w_{h^{2}} q_{h}^{2}+\sum_{h_{1}=1, h_{1}<h_{2}}^{n} w_{h_{1} h_{2}} q_{h_{1}} q_{h_{2}}
$$

Compared it with Taguchi's model, we can see that (2) is actually the extended multivariate form of Taguchi's quality loss model.

Here, Qproduct is a target total quality index and described as the quality loss. All the n partial quality indices influence Qproduct in a coaction mode, and qh is the relative quality deviant. Every partial quality deviant can be gotten by statistic. Here we use the relative partial quality deviant, and it can be expressed by the relative quality value of the deviant from the target value and severalfold tolerances. If the value of quality characteristic lies beyond the tolerance, it should be multiplied by a punishment factor.

where, $\sum_{h=1}^{H} w_{h^{2}} q_{h}^{2}, \sum_{h_{1}=1, h_{1}<h_{2}}^{n} w_{h_{1} h_{2}} q_{h_{1}} q_{h_{2}}$ are separately the self-action item and the inter-action item of the partial quality deviants. $w_{h^{2}}$ is the self-action influence weight of the quality deviant $q_{h}^{2} ; w_{h_{1} h_{2}}$ is the inter-action weight of deviant $q_{h_{1}} q_{h_{2}}$.

Compared with our expansion equation, it can be gotten

$$
\begin{aligned}
& \quad w_{h^{2}}=\frac{1}{2 !} f_{Q_{h}^{2}}^{\prime \prime}(0, \ldots, 0, \ldots 0)=\frac{1}{2} f_{Q_{h}^{2}}^{\prime \prime}(0, \ldots, 0, \ldots 0) \\
& w_{h_{1} h_{2}}=\frac{1}{2 !} f_{Q_{h_{1}} Q_{h_{2}}}^{\prime \prime}(0, \ldots, 0, \ldots 0) \\
& =\frac{1}{2}\left[f_{Q_{h_{1}} Q_{h_{2}}}^{\prime \prime}(0, \ldots, 0, \ldots 0)+f_{Q_{h_{2}} Q_{h_{1}}}^{\prime \prime}(0, \ldots, 0, \ldots 0)\right] \\
& =f_{Q_{h_{1}} Q_{h_{2}}}^{\prime \prime}(0, \ldots, 0, \ldots 0)
\end{aligned}
$$

If necessary, we can also expand the product quality model further more to U-order. 
$Q_{\text {product }}=\sum_{u=2}^{U}\left\{\frac{1}{u !} \sum_{h_{h_{1}}, h_{2}, \ldots h_{u}=1}^{H}\left[f^{(u)}{ }_{Q_{m_{1}} Q_{h_{2}} \ldots Q_{h_{u}}}(0,0 \ldots, 0) q_{h_{1}} q_{h_{2}} \ldots q_{h_{u}}\right]\right\}+R_{U}$

$R_{U}=o\left(\rho^{U}\right)$

When the quality model is expanded to 2-order, the inter-actions of partial quality indices have been fully considered. In fact, the impactions of items higher than 2 -order are very little $[10,11]$. Thus for the simplification of computation and the actual requirement, further higher orders are never needed.

The above work suits the small-is-better and the nominal-is-best problems. The large-is-better problem can also be reformed into a small-is-better problem.

\section{Coefficient Determination}

\subsection{The Tolerance Limits Method}

The quality loss model (2) can also be written as

$$
Q_{\text {product }}=\sum_{i=1}^{H} w_{i i} q_{i}^{2}+\sum_{i=1, i<j}^{n} w_{i j} q_{i} q_{j}
$$

the coefficients $w_{i j}$ can be determined using the following method all these $w_{i j}(i \leq j)$ can constitute a upper triangular matrix like Figure 1.

1) Determine the elements in Diagonal for an arbitrary $i(i=1,2, \ldots, H)$, when all the $q_{k}=0,(k \neq i) \Delta_{i}$ is the tolerance limit of defective for self quality and $A_{i}$ is the self quality loss when defective happens.

Then we have

$$
Q_{\text {product }}=A_{i}=\sum_{i=1}^{H} w_{i i} q_{i}^{2}+\sum_{i=1, i<j}^{n} w_{i j} q_{i} q_{j}=w_{i i}\left(\Delta_{i}\right)^{2}
$$

So

$$
w_{i i}=A_{i} /\left(\Delta_{i}\right)^{2}
$$

2) Determine other elements for an arbitrary $i, j$ $(i, j=1,2, \ldots, H, i \leq j)$, when all the $q_{k}=0,(k \neq i, k \neq j), \Delta_{i j}^{i}$ is the tolerance limit of quality $i$ for coaction quality $i$ and $j, \Delta_{i j}^{j}$

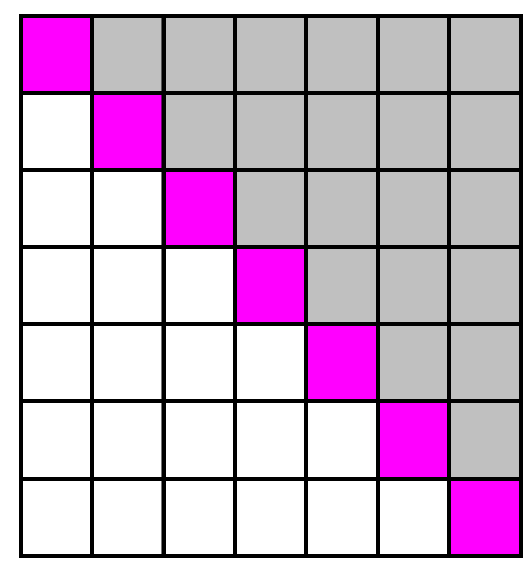

Figure 1. Upper triangular matrix of coefficients then, we can determine these coefficients is the tolerance limit of quality $j$ for coaction quality $i$ and $j$ and $A_{i j}$ is the coaction quality loss when coaction defective happens.

Then we have

$$
\begin{aligned}
& Q_{\text {product }}=A_{i j}^{0}=\sum_{i=1}^{H} w_{i i} q_{i}^{2}+\sum_{i=1, i<j}^{n} w_{i j} q_{i} q_{j} \\
& =w_{i i}\left(\Delta_{i j}^{0, i}\right)^{2}+w_{j j}\left(\Delta_{i j}^{0, j}\right)^{2}+w_{i j} \Delta_{i j}^{0, i} \Delta_{i j}^{0, j}
\end{aligned}
$$

Thus we have

$$
w_{i j}=\left[A_{i j}^{0}-w_{i i}\left(\Delta_{i j}^{0, i}\right)^{2}-w_{j j}\left(\Delta_{i j}^{0, j}\right)^{2}\right] / \Delta_{i j}^{0, i} \Delta_{i j}^{0, j}
$$

where

$$
w_{i i}=A_{i}^{0} /\left(\Delta_{i}^{0}\right)^{2}, \quad w_{j}=A_{j}^{0} /\left(\Delta_{j}^{0}\right)^{2}
$$

This coefficient determination process can be described in Figure 2.

Remark:

In some particular circumstances,

$$
\Delta_{i j}^{0, i}=\Delta_{i}^{0} \quad, \quad \Delta_{i j}^{0, j}=\Delta_{j}^{0}
$$

we have a simplified form

$$
w_{i j}=\left[A_{i j}^{0}-A_{i}^{0}-A_{j}^{0}\right] / \Delta_{i}^{0} \Delta_{j}^{0}
$$

\subsection{Other Methods}

To determinate the weight coefficients, the least square method can also be adopt. However linear neural networks are more suitable [11-14]. The input weights of the trained neural network are just the weight coefficients of the model.

\section{Conclusions}

Based on the quality loss model of Taguchi, the total quality model which is based on multivariate relative quality deviants was studied. A 2-order product quality model, which was based on several indices of relatively quality deviation, was built. Then, the model was successfully extended to 3 or even higher orders. To deduce

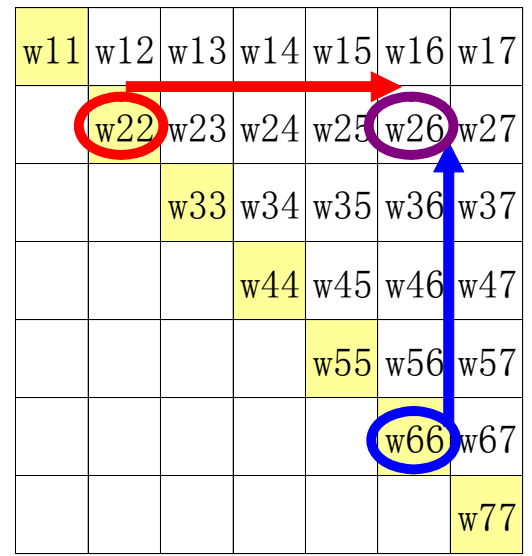

Figure 2. From the diagonal elements to other elements 
a simplified formula, a new simplified product quality model (a multivariate quality loss model) was built and the error of it was also analyzed. Finally, a useful method of the coefficient determination was brought forward to determinate all the coefficients in the multivariate quality loss model and the work flow of it was also described.

However, in the complex product, the coupling factor, structure layer and the number of parts may have a further increase, and the quality loss model become more and more complex. Then there are no enough data for the determination of the coefficients and the calculation also become more and more complex. To make full use of historical data and similar data, a quick-response incremental model of multivariate quality loss is needed. So it should be in our further research.

\section{Acknowledgment}

This project was supported by National Natural Science Foundation of China (70801036), Natural Science Foundation of the Jiangsu Higher Education Institutions of China (07KJB460045).

\section{REFERENCES}

[1] G. Taguchi, "Quality engineering [M]," China Translation and Publishing Corporation, December 1985.

[2] H. J. Powell, "Quality tools for high value fabrications [J]," Welding in the World, No. 46 (SPEC), pp. 275-280, July 2002.

[3] R. Rai and V. Allada, "Modular product family design: Agent-based pareto-optimization and quality loss function-based post-optimal analysis [A]," International Journal of Production Research, Vol. 41, No. 17, pp. 40754098, November 20, 2003.

[4] S. H. Fan, "Studies on Grey theory based intelligent methods and their applications to MCMQC [D],"
Tsinghua University, Beijing, June 2004.

[5] W. M. Chan, R. N. Ibrahim, and P. B. Lochert, "Quality evaluation model using loss function for multiple S-type quality characteristics [J]," International Journal of Advanced Manufacturing Technology, Vol. 26, No. 1-2, pp. 98-101, 2005

[6] R. Khorramshahgol and G. R. Djavanshir, "The application of analytic hierarchy process to determine proportionality constant of the Taguchi quality loss function [J]," IEEE Transactions on Engineering Management, , Vol. 55, No. 2, pp. 340-348, May 2008.

[7] S. H. Fan, "The coefficient determination of multivariate quality loss model,” IEEE EMS’07, 2007.

[8] G. X. Zhang, "Quality management [M]," Higher Education Press, Beijing, 1998.

[9] S. H. Fan, T. Y. Xiao, et al., "Building the dynamic network of mass customization [J]," Journal of System Simulation, Vol. 16, No. 7, pp. 1597-1599, 2004

[10] S. H. Fan, T. Y. Xiao, et al., "Model building of the dynamic network in MCMQA [J]," Journal of System Simulation, Vol. 16, No. 12, pp. 2767-2769, 2004.

[11] P. Melin and O. Castillo, "A general method for surface quality control in intelligent manufacturing of materials using a new fuzzy-fractal approach fuzzy [A]," Information Processing Society, NAFIPS, 18th International Conference of the North American, pp. 10-12, June 1999.

[12] M. Q. Zhu, "Artificial neural network approach of quality control for intelligent manufacturing system $[\mathrm{J}]$," Acta Aeronautica et Astronautica Sinica, Vol. 18, No. 5, pp. 603-606, 1997.

[13] S. Fan, "Studies on the application of grey genetic algorithm in dynamic network of MCMQA," Mechanical Science and Technology, Vol. 25, No. 8, pp. 984-988, August 2006.

[14] S. Fan, "E-factory based agile-maintenance simulationplatform," Journal of System Simulation, Vol. 19, No. 5, pp. 1151-1153, May 2007. 